\title{
Patterns of recurrence in patients with curative resected rectal cancer according to different chemoradiotherapy strategies: Does preoperative chemoradiotherapy lower the risk of peritoneal recurrence?
}

\author{
HYUNGWOO $\mathrm{CHO}^{1 *}$, JEONG EUN KIM ${ }^{1 *}$, SUN YOUNG KIM ${ }^{1}, \mathrm{KYU}^{*}$ YYO KIM ${ }^{1}$, TAE WON KIM ${ }^{1}$, \\ JIN-HONG PARK ${ }^{2}$, JONG HOON KIM ${ }^{2}$, SEOK-BYUNG LIM ${ }^{3}$, CHANG SIK YU ${ }^{3}$, \\ JIN CHEON KIM ${ }^{3}$ and YONG SANG HONG ${ }^{1}$
}

Departments of ${ }^{1}$ Oncology, ${ }^{2}$ Radiation Oncology and ${ }^{3}$ Surgery, Asan Medical Center, University of Ulsan College of Medicine, Seoul 05505, Republic of Korea

Received May 18, 2020; Accepted August 10, 2020

DOI: $10.3892 / \mathrm{ol} .2020 .12105$

\begin{abstract}
The present study aimed to compare the pattern of distant recurrence between patients with non-metastatic rectal cancer treated with pre-operative (OP) and those treated with post-operative (post-OP) chemoradiotherapy (CRT). A total of 631 patients with newly diagnosed non-metastatic rectal cancer who had received pre-OP or post-OP CRT with curative intent surgery between August 2008 and April 2015 were identified. Inverse probability of treatment weighting (IPTW) was performed to account for baseline differences between the two arms. Overall, 449 and 182 patients were treated with pre-OP and post-OP CRT, respectively. Sex, tumor location, clinical tumor stage, CRT regimen and adjuvant chemotherapy regimen were significantly different between the two arms. The median follow-up duration was 55.4 months (range, 53.7-57.1). The 5-year distant recurrence-free survival (RFS) rates and
\end{abstract}

Correspondence to: Dr Yong Sang Hong, Department of Oncology, Asan Medical Center, University of Ulsan College of Medicine, 88 Olympic-ro 43-gil, Songpa-gu, Seoul 05505, Republic of Korea

E-mail: yshong@amc.seoul.kr

*Contributed equally

Abbreviations: CI, confidence interval; CRT, chemoradiotherapy; CT, computed tomography; DFS, disease-free survival; ECOG PS, Eastern Cooperative Oncology Group Performance Status; HR, hazard ratio; IPTW, inverse probability of treatment weighting; OS, overall survival; post-OP, post-operative; pre-OP, pre-operative; PS, propensity score; RFS, recurrence-free survival; TME, total mesorectal excision

Key words: rectal neoplasms, pre-operative chemoradiotherapy, post-operative chemoradiotherapy, recurrence pattern, peritoneal recurrence 5-year overall survival (OS) rates were not significantly different between the pre-OP and post-OP CRT arms (RFS, 67.5 vs. $71.6 \%, \mathrm{P}=0.595$ and $\mathrm{OS}, 81.9$ vs. $77.0 \%, \mathrm{P}=0.449)$, and no difference was observed in the distant recurrence patterns. Following IPTW, there was still no difference in distant RFS (pre-OP vs. post-OP CRT; hazard ratio $(\mathrm{HR})=0.62 ; \mathrm{P}=0.911$ ), but pre-OP CRT was significantly associated with lower peritoneal recurrence (pre-OP vs. post-OP CRT; HR, 0.13; P=0.032). In addition, there was no significant difference in OS between the two arms (pre-OP vs. post-OP CRT; HR, 0.85; $\mathrm{P}=0.665$ ). In conclusion, although distant RFS was not significantly different between the two arms, pre-OP CRT was significantly associated with a lower risk of peritoneal recurrence than post-OP CRT in patients non-metastatic rectal cancer.

\section{Introduction}

Colorectal cancer is the third most common type of cancer and was the second leading cause of cancer-associated mortality worldwide in 2015 , with rectal cancer accounting for $\sim 40 \%$ of these mortalities (the number of deaths from colon cancer was 551,269 and the number of deaths from rectal cancer was 310,394) (1). While the treatment approaches for metastatic colon and rectal cancer are similar, treatment approaches for resectable cancer vary according to the affected organ.

The adoption of multimodal therapy that combines chemoradiotherapy (CRT) and total mesorectal excision (TME) has led to more efficient local disease control and improved survival for patients with non-metastatic rectal cancer (2-5). Previous studies have suggested that pre-operative (pre-OP) CRT has advantages over post-operative (post-OP) CRT in terms of treatment compliance, safety, and local control $(6,7)$. Accordingly, the current standard course of treatment involves pre-OP CRT followed by TME, particularly in cases of locally advanced disease. However, pre-OP CRT has not led to improvements in rates of distant recurrence and overall survival $(7,8)$. Furthermore, patients with early-stage tumors that do not require radiotherapy may be overtreated with 
pre-OP CRT (8). In addition, potential disadvantages of pre-OP CRT include the development of distant metastasis during the pre-OP CRT period and the resting period until surgery, thereby missing the opportunity for curative resection (9). For these reasons, a subpopulation of rectal cancer patients continues to receive upfront surgery followed by post-OP CRT (10).

Several preclinical studies have suggested that radiotherapy may induce changes in the tumor microenvironment, including dysfunction of the endothelial cells of the tumor vasculature and epithelial-to-mesenchymal transition in tumor cells, which may promote tumor invasion and spread $(11,12)$. Furthermore, it is well-known that histological subtype influences metastatic patterns; therefore, radiation-induced biological changes within the primary tumor prior to resection may also affect the distribution of metastatic spread (13). Although the incidence of distant recurrence is not different between pre-OP and post-OP CRT, the pattern of distant relapse may be affected by the timing of CRT. A recent study suggested that pre-OP CRT in patients with rectal cancer may affect the pattern of recurrence (14). However, to date, to the best of our knowledge no studies have compared the pattern of distant relapse between patients treated with pre-OP and those treated with post-OP CRT. Understanding the patterns of distant recurrence following initial treatment may aid clinicians in improving the monitoring of patients with resectable rectal cancer.

Therefore, the present study analyzed and compared patterns of distant recurrence in patients with non-metastatic rectal cancer according to treatment with pre-OP or post-OP CRT.

\section{Materials and methods}

Patients. The present study identified and retrospectively reviewed the medical records of patients with histologically-confirmed non-metastatic rectal cancer who received pre-OP or post-OP CRT with curative intent surgery at Asan Medical Center (Seoul, South Korea) between August 2008 and April 2015. Patients were excluded if they had metastatic disease prior to or at the time of surgery. The following patient data were extracted from the medical records for analysis: Age, sex, Eastern Cooperative Oncology Group Performance Status (ECOG PS) (15), tumor pathology (16), clinical stage (17), tumor location relative to the anal verge, neoadjuvant CRT regimen (18), adjuvant chemotherapy regimen (19), and initial site(s) of recurrence. The protocols of the present study were approved by the Institutional Review Board at Asan Medical Center (AMC IRB 2008-0256), which waived the requirement for informed consent.

Treatment and response assessment. Pre-OP or post-OP CRT was selected for patients following consultation by a multidisciplinary team that included a medical oncologist, surgeon, and radiation oncologist. For patients who received pre-OP CRT, surgery was performed within 4-6 weeks after completion of CRT. With the exception of laparoscopic trans-anal excision surgery in a subpopulation of patients, all surgeries were performed using TME (4). Adjuvant chemotherapy was initiated between 3 and 8 weeks after surgery or immediately after completion of post-OP CRT. Following the completion of adjuvant chemotherapy, chest radiography and carcinoembryonic antigen measurements were performed every 3 months for the first 2 years and every 6 months thereafter. Computed tomography (CT) scans were performed every 6 months for the abdominopelvic region and annually for the chest region. Colonoscopic assessments were performed at 1,3 and 5 years after surgery.

Statistical analyses. All time-to-event endpoints were calculated from the start date of CRT for patients who underwent pre-OP CRT and from the date of surgery for patients who underwent post-OP CRT. Local recurrence was defined as clinically-confirmed relapse within the perineum or pelvis. Distant recurrence was defined as relapse in sites other than the perineum or pelvis. Disease-free survival (DFS) was calculated from the start date of CRT (pre-OP CRT arm) or surgery (post-OP CRT arm) until tumor relapse. Local and distant recurrence-free survival (RFS) rates were calculated from the start date of CRT (pre-OP CRT arm) or surgery (post-OP CRT arm) until local or distant recurrence, respectively. Overall survival (OS) was calculated as the time from the start date of CRT (pre-OP CRT arm) or surgery (post-OP CRT arm) until mortality from any cause. The Kaplan-Meier method was used to calculate DFS, local/distant RFS, and OS, and comparisons were analyzed using the log-rank test.

Baseline characteristics and recurrence patterns were compared between the arms using the Student's t-test for continuous variables and the Fisher's exact test or Pearson's Chi-square test for categorical variables, as appropriate. To reduce the impact of treatment selection bias and potential confounding, adjustment for significant differences in baseline characteristics of patients was performed using weighted Cox proportional hazards regression models using inverse probability of treatment weighting (IPTW) and robust standard errors $(20,21)$. The propensity score (PS) to receive pre-OP vs. post-OP CRT was estimated using a multivariable logistic regression model based on age, ECOG PS, sex, clinical stage, tumor location from the anal verge, tumor histology, CRT regimen, and adjuvant chemotherapy regimen. The PS was estimated without accounting for outcomes. Tumor histology, CRT regimen, and adjuvant chemotherapy regimen were included in the IPTW analysis since it has been reported that variables associated with outcomes of interest should be included even if they are unrelated to treatment selection to further reduce bias in the PS model $(20,22)$. IPTW for patients treated with pre-OP CRT were the inverse of PS, and IPTW for patients treated with post-OP CRT were the inverse of 1-PS. The treatment effect of pre-OP vs. post-OP CRT was estimated using weighted Cox proportional hazards regression models with IPTW and robust standard errors.

All statistical analyses were performed using SAS version 9.4 (SAS Institute Inc.). P $<0.05$ was considered to indicate a statistically significant difference.

\section{Results}

Patient characteristics. A total of 631 patients with newly diagnosed non-metastatic rectal cancer who received pre-OP CRT $(n=449)$ or post-OP CRT $(n=182)$ with curative intent surgery were identified between August 2008 and April 2015. The median total irradiation dose was $50 \mathrm{~Gy}$ (range, 6-55 Gy). 
Table I. Baseline characteristics of patients who received pre-OP CRT vs. post-OP CRT.

\begin{tabular}{|c|c|c|c|c|c|c|}
\hline \multirow[b]{2}{*}{ Characteristic } & \multicolumn{3}{|c|}{ Before IPTW } & \multicolumn{3}{|c|}{ After IPTW } \\
\hline & $\begin{array}{c}\text { Pre-OP CRT } \\
\text { n }(\%) \\
(n=449)\end{array}$ & $\begin{array}{c}\text { Post-OP CRT } \\
\mathrm{n}(\%) \\
(\mathrm{n}=182)\end{array}$ & P-value ${ }^{a}$ & $\begin{array}{c}\text { Pre-OP CRT } \\
\text { n (\%) } \\
(\mathrm{n}=634)\end{array}$ & $\begin{array}{c}\text { Post-OP CRT } \\
\mathrm{n}(\%) \\
(\mathrm{n}=520)\end{array}$ & P-value ${ }^{b}$ \\
\hline Mean age, years \pm SD & $57.6 \pm 11.1$ & $58.7 \pm 10.1$ & 0.24 & $57.6 \pm 12.9$ & $60.0 \pm 18.5$ & 0.22 \\
\hline Sex & & & 0.006 & & & 0.38 \\
\hline Female & $143(31.8)$ & $79(43.4)$ & & $228(35.9)$ & $223(42.9)$ & \\
\hline Male & $306(68.2)$ & $103(56.6)$ & & $406(64.1)$ & $297(57.1)$ & \\
\hline ECOG-PS & & & 0.20 & & & 0.64 \\
\hline $0-1$ & $448(99.8)$ & $180(98.9)$ & & $632(99.6)$ & $517(99.4)$ & \\
\hline 2 & $1 \quad(0.2)$ & $2(1.1)$ & & $2(0.4)$ & $3(0.6)$ & \\
\hline Location from AV & & & $<0.001$ & & & 0.75 \\
\hline $0-4 \mathrm{~cm}$ & $209(46.5)$ & $19(10.4)$ & & $232(36.5)$ & $165(31.8)$ & \\
\hline$>4 \mathrm{~cm}, \leq 8 \mathrm{~cm}$ & $207(46.1)$ & 109 (59.9) & & $310(48.9)$ & $263(50.5)$ & \\
\hline$>8 \mathrm{~cm}$ & $33 \quad(7.3)$ & $54(29.7)$ & & $92(14.6)$ & $92(17.8)$ & \\
\hline Clinical tumor stage & & & $<0.001$ & & & 0.43 \\
\hline $0-2$ & $27 \quad(6.0)$ & $32(17.6)$ & & $49 \quad(7.7)$ & $52(10.1)$ & \\
\hline $3-4$ & $422(94.0)$ & $150(82.4)$ & & $585(92.3)$ & $468(89.9)$ & \\
\hline Clinical node stage & & & 0.26 & & & 0.82 \\
\hline- & $52(11.6)$ & $27(14.8)$ & & $68(10.8)$ & $52(10.1)$ & \\
\hline+ & $397(88.4)$ & $155(85.2)$ & & $566(89.2)$ & 468 (89.9) & \\
\hline Clinical TNM stage & & & 0.17 & & & 0.96 \\
\hline I-II & $49(10.9)$ & $27(14.8)$ & & $65(10.3)$ & $52(10.1)$ & \\
\hline III & $400(89.1)$ & $155(85.2)$ & & $569(89.7)$ & 468 (89.9) & \\
\hline High grade histology & & & 0.06 & & & 0.11 \\
\hline $\mathrm{No}^{\mathrm{c}}$ & $414(92.2)$ & 177 (97.3) & & $594(93.7)$ & $502(96.5)$ & \\
\hline Yes $^{d}$ & $30 \quad(6.7)$ & $5 \quad(2.7)$ & & $34 \quad(5.4)$ & $18 \quad(3.5)$ & \\
\hline Undetermined & $5 \quad(1.1)$ & $0 \quad(0.0)$ & & $5 \quad(0.9)$ & $0 \quad(0.0)$ & \\
\hline CRT regimen & & & $<0.001$ & & & 0.86 \\
\hline 5-FU/LV & $307(68.4)$ & $162(89.0)$ & & $468(73.7)$ & $390(75.0)$ & \\
\hline Capecitabine & $117(26.1)$ & $16(8.8)$ & & $133(20.9)$ & $109(20.9)$ & \\
\hline Other & 19 (4.2) & 3 (1.6) & & $26 \quad(4.1)$ & $19 \quad(3.6)$ & \\
\hline Unknown & $6 \quad(1.3)$ & $1 \quad(0.5)$ & & $8 \quad(1.2)$ & $3(0.6)$ & \\
\hline Adjuvant chemotherapy regimen & & & $<0.001$ & & & 0.003 \\
\hline 5-FU/LV & $285(63.5)$ & 143 (78.6) & & $431(67.9)$ & $372(71.5)$ & \\
\hline FOLFOX & $87(19.4)$ & 3 (1.6) & & $91(14.3)$ & $22(4.3)$ & \\
\hline Capecitabine & $15(3.3)$ & $15(8.2)$ & & $33 \quad(5.2)$ & $29 \quad(5.6)$ & \\
\hline Other & $25(5.6)$ & 3 (1.6) & & $28(4.4)$ & $7 \quad(1.3)$ & \\
\hline None & $37(8.2)$ & 18 (9.9) & & $52 \quad(8.2)$ & $90(17.2)$ & \\
\hline
\end{tabular}

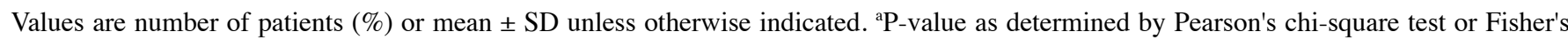
exact test for categorical variables and Student's t-test for continuous variables as appropriate. ${ }^{b} \mathrm{P}$-value as determined by generalized estimating


erative; CRT, chemoradiotherapy; post-OP, postoperative; IPTW, inverse probability of treatment weighting; SD, standard deviation; ECOG PS, Eastern Cooperative Oncology Group Performance Status; AV, anal verge; 5-FU, fluorouracil; LV, leucovorin; FOLFOX, 5-FU/LV plus oxaliplatin; TNM, tumor-node-metastasis.

Patient baseline characteristics are summarized in Table I. Age, ECOG PS, clinical stage, tumor histology, and CRT regimen were similar between the two arms. The pre-OP CRT arm had a higher proportion of male patients ( 68.2 vs. $56.6 \%, \mathrm{P}=0.006)$, had a tumor location closer to the anal verge (distance from anal verge, $\leq 4 \mathrm{~cm} ; 46.5$ vs. $10.4 \%, \mathrm{P}<0.001$ ), and had higher clinical T stages (stage $3-4 ; 94.0$ vs. $82.4 \%$; $\mathrm{P}<0.001$ ) than patients in the post-OP CRT arm. The two arms did not show 
Table II. Initial distant recurrence pattern.

\begin{tabular}{|c|c|c|c|c|}
\hline Recurrence pattern & $\begin{array}{c}\text { Total } \\
\mathrm{n}(\%) \\
(\mathrm{n}=631)\end{array}$ & $\begin{array}{c}\text { Pre-OP CRT } \\
\text { n (\%) } \\
(\mathrm{n}=449)\end{array}$ & $\begin{array}{c}\text { Post-OP CRT } \\
\text { n (\%) } \\
(\mathrm{n}=182)\end{array}$ & P-value \\
\hline \multicolumn{5}{|l|}{ Distant metastasis } \\
\hline Liver & $54(8.6)$ & $38 \quad(8.5)$ & $16(8.8)$ & 0.89 \\
\hline Lung & $113(17.9)$ & $83(18.5)$ & $30(16.5)$ & 0.55 \\
\hline Distant lymph node & $47(7.4)$ & $38(8.5)$ & $9(4.9)$ & 0.13 \\
\hline Bone & $8(1.3)$ & $6(1.3)$ & $2(1.1)$ & 1.00 \\
\hline Peritoneum & 10 (1.6) & $6(1.3)$ & $4 \quad(2.2)$ & 0.49 \\
\hline Other $^{\mathrm{a}}$ & $12(1.9)$ & $10(2.2)$ & $2(1.1)$ & 0.52 \\
\hline Sum of distant metastasis & & & & 0.93 \\
\hline 1 organ & $150(23.8)$ & $110(24.5)$ & $40(22.0)$ & \\
\hline$\geq 2$ organs & $41(6.5)$ & $31(6.9)$ & $10(5.5)$ & \\
\hline
\end{tabular}

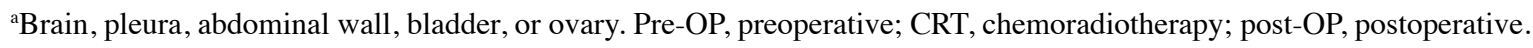
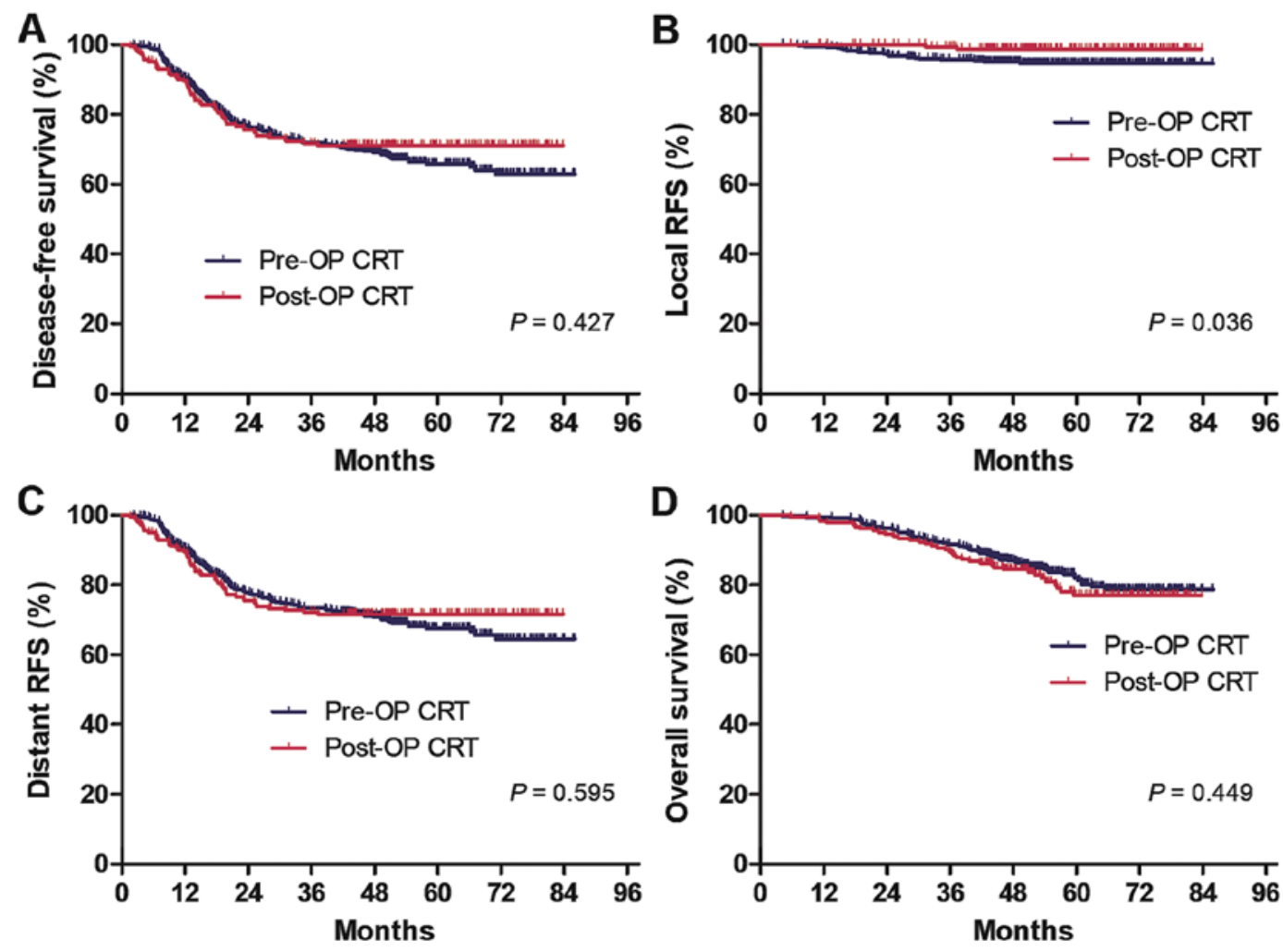

Figure 1. Survival outcomes in the unweighted cohort. (A) Disease-free survival, (B) local RFS, (C) distant RFS, and (D) overall survival rates. RFS, recurrence-free survival; Pre-OP, preoperative; Post-OP, postoperative; CRT, chemoradiotherapy.

significant differences in terms of CRT regimen or adjuvant chemotherapy regimens.

Survival analysis in the unweighted cohort. With a median follow-up time of 55.4 months (range, 53.7-57.1), the 5-year DFS and OS rates of all the patients together were 65.1 and $80.4 \%$, respectively. In decreasing order of frequency, distant recurrence occurred in the lung (17.9\%), the liver (8.6\%), and distant lymph nodes (7.4\%; Table II). Single-organ distant recurrence was observed in 150 patients (23.8\%) and multiple-organ distant recurrence in 41 patients (6.5\%). The 5-year DFS rates for the pre-OP and post-OP CRT arms were 65.8 and $71.0 \%$, respectively ( $\mathrm{P}=0.43$; Fig. 1A). The 5-year local RFS rates was significantly lower in the pre-OP CRT arm than in the post-OP CRT arm (94.5 vs. $98.8 \%$; P=0.04; Fig. 1B), but no significant difference was observed in the 5-year distant RFS rates (pre-OP vs. post-OP CRT; 67.5 vs. $71.6 \%$; $\mathrm{P}=0.60$; Fig. $1 \mathrm{C}$ ). The 5-year OS rate was $81.9 \%$ in the pre-OP CRT arm and 
Table III. IPTW-adjusted estimated HR of preoperative CRT on DFS, local/distant RFS, OS, and sites of distant recurrence.

\begin{tabular}{llc}
\hline & \multicolumn{1}{c}{ IPTW analysis $^{\mathrm{a}}$} \\
\cline { 2 - 3 } Outcome variables & \multicolumn{1}{c}{ HR $(95 \% \mathrm{CI})$} & P-value \\
\hline DFS & $1.07(0.65-1.78)$ & 0.79 \\
OS & $0.86(0.42-1.73)$ & 0.67 \\
Local RFS & $4.39(0.86-22.37)$ & 0.08 \\
Distant RFS & $1.03(0.62-1.72)$ & 0.91 \\
Sites of distant recurrence & & \\
Liver & $0.60(0.23-1.59)$ & 0.31 \\
Lung & $1.50(0.84-2.67)$ & 0.17 \\
Distant lymph node & $1.21(0.35-4.21)$ & 0.76 \\
Bone & $0.26(0.02-2.82)$ & 0.27 \\
Peritoneum & $0.13(0.02-0.84)$ & 0.03 \\
Other & $3.68(0.73-18.54)$ & 0.11 \\
\hline
\end{tabular}

${ }^{a}$ Using robust sandwich variance estimator. IPTW, inverse probability of treatment weighting; HR, hazard ratio; CRT, chemoradiotherapy; DFS, disease-free survival; RFS, recurrence-free survival, OS, overall survival; $\mathrm{CI}$, confidence interval.

$77.0 \%$ in the post-OP CRT arm ( $\mathrm{P}=0.45$; Fig. 1D). Distant recurrence patterns were not significantly different between the two arms (Table II).

IPTW analysis. Following IPTW-adjustment, the baseline characteristics of the two arms were well-balanced, with the exception of the adjuvant chemotherapy regimen (Table I), which was additionally adjusted for using regression for further analysis in the weighted cohort. Table III summarizes the IPTW-adjusted estimated hazard ratios (HRs) for DFS, $\mathrm{OS}$, and recurrence of patients who received pre-OP CRT, compared with those who received post-OP CRT. There were no significant differences between the two arms in DFS [HR, 1.07; 95\% confidence interval (CI), 0.65-1.78; $\mathrm{P}=0.79$ ], local RFS (HR, 4.38; 95\% CI, 0.86-22.37; $\mathrm{P}=0.08$ ), or distant RFS (HR, 0.62; 95\% CI, 0.62-1.72; P=0.91). The distant recurrence patterns were similar between the two arms, but pre-OP CRT was associated with a significantly lower risk of peritoneal recurrence ( $\mathrm{HR}, 0.13 ; 95 \% \mathrm{CI}, 0.02-0.84 ; \mathrm{P}=0.03$ ). The OS was not significantly different between the two arms (HR, 0.85; 95\% CI, 0.42-1.73; P=0.67).

\section{Discussion}

Although it has been suggested that the cumulative incidence of distant recurrence in patients with resectable rectal cancer is not significantly different between patients who receive pre-OP or post-OP CRT $(6,7)$, the patterns of distant recurrence following pre-OP or post-OP CRT have not been adequately compared. To the best of our knowledge, the present study was the first to evaluate and compare the distant recurrence patterns of patients with non-metastatic rectal cancer treated with either pre-OP or post-OP CRT. After performing IPTW-adjustment to balance the baseline characteristics, there was no significant difference in distant RFS, but peritoneal recurrence was significantly lower in patients treated with pre-OP CRT.

As previously reported, TME and CRT have significantly improved local control of rectal cancer, with patients with locally advanced rectal cancer showing a cumulative local recurrence rate of $<10 \%(6,7,23)$. However, distant recurrence occurs in $20-50 \%$ of patients, with the lungs and the liver being the most frequent sites of metastasis $(6,7,14,23)$. Similar results were observed in the present study with 5-year local DFS rates of 94.5 and $98.8 \%$ and 5-year distant RFS rates of 67.5 and $71.6 \%$ for the pre-OP and post-OP CRT arms, respectively. In addition, in the entire cohort of the present study, the lungs $(17.9 \%)$ were the most common distant recurrence site, followed by the liver $(8.6 \%)$.

In the unweighted cohort, the pre-OP and post-OP CRT arms did not have significantly different distant recurrence patterns. However, following IPTW adjustment, pre-OP CRT was associated with a significantly lower risk of peritoneal metastasis (vs. post-OP CRT; HR, 0.13; P=0.03). Although this result should be cautiously interpreted due to the low overall incidence of peritoneal recurrence in the present study, it is consistent with the findings observed in a recent study, where pre-OP radiotherapy was negatively associated with the risk of peritoneal carcinomatosis (24). It has been reported that radiotherapy causes an increase in the number of fibroblasts and a decrease in the number of immune cells within the tumor microenvironment (25). Furthermore, radiotherapy is also associated with an increase in the mucinous component within tumors (26). Although the mechanism driving the association between radiotherapy-induced changes in the tumor microenvironment and peritoneal metastasis is unclear, the characteristics of a tumor with a predilection for peritoneal metastasis in certain patients may be changed by radiotherapy, leading to a reduction in the likelihood of peritoneal metastasis.

In the unweighted cohort, the pre-OP CRT arm had a significantly lower 5-year local RFS rate than the post-OP CRT arm (94.5 vs. $98.8 \%$; $\mathrm{P}=0.04)$. This finding contradicts the results of previous studies where pre-OP CRT had significantly improved local control than post-OP CRT $(6,7)$. Discrepancies between the results of previous studies and those of the present study may be due to a higher proportion of patients with a tumor location closer to the anal verge $(\leq 4 \mathrm{~cm})$ in the pre-OP CRT arm (pre-op vs. post-OP CRT; 46.5 vs. $10.4 \%$; $\mathrm{P}<0.001)$. It has been suggested in previous studies that distant rectal cancer has higher local recurrence rates $(23,27)$. After performing IPTW-adjustment, a significant difference in the local RFS rates between the two arms was not observed $(\mathrm{P}=0.08)$. In line with the results of previous studies, the two arms did not show significant differences in terms of distant RFS and OS rates between the unweighted and weighted cohorts $(6,7)$.

There are several limitations of the present study. As anticipated for any retrospective study, selection bias may exist. A considerable number of baseline characteristics were significantly different between the two arms. To minimize the impact of selection bias, weighted Cox proportional hazards regression modeling with IPTW was utilized. The IPTW approach is commonly used to control for confounding variables in observational studies of medical interventions, which uses the 
entire cohort and may address a large number of confounding variables. However, IPTW estimates may be highly unstable in the presence of large weights because the estimates may be driven by outcomes occurring in a small number of patients. In addition, differences in adjuvant chemotherapy regimens remained after IPTW, which is likely due to the small number of patients in the post-OP CRT arm, and therefore required additional adjustment. Furthermore, the effect of pre-OP CRT on the tumor microenvironment has not been evaluated, and a comparative analysis of pre-OP and post-OP CRT tumor specimens regarding histopathological or immunological changes and gene expression profiles is necessary to better understand the distant recurrence patterns. Furthermore, the median follow-up duration in the present study was relatively short. Despite these limitations, the present study is the first to directly evaluate and compare distant recurrence patterns in a large number of patients with non-metastatic rectal cancer treated with pre-OP or post-OP CRT.

The results of the present study demonstrated that, although there was no difference in distant RFS between patients who underwent pre-OP or post-OP CRT, after IPTW analysis, pre-OP CRT was associated with a lower risk of peritoneal recurrence in patients with non-metastatic rectal cancer, compared with post-OP CRT. Further study is required to evaluate the effect of CRT on the tumor microenvironment and the corresponding association with the recurrence pattern.

\section{Acknowledgements}

Not applicable.

\section{Funding}

The present study was supported by a grant from the Korea Health Technology R\&D Project through the Korea Health Industry Development Institute (KHIDI), funded by the Ministry of Health \& Welfare, Republic of Korea (grant no. HI18C2383).

\section{Availability of data and materials}

The datasets used and/or analyzed during the current study are available from the corresponding author upon reasonable request.

\section{Authors' contributions}

HC and JEK contributed toward the acquisition, analysis, and interpretation of data; and drafted the manuscript. KPK, SYK, YSH and TWK contributed toward the conception and design of the study and interpretation of data, and critically revised the manuscript. JHP, JHK, SBL, CSY and JCK contributed toward the acquisition of data and critically revised the manuscript. All authors read and approved the final manuscript.

\section{Ethics approval and consent to participate}

The protocols of the present study were approved by the Institutional Review Board at Asan Medical Center (grant no. AMC IRB 2008-0256), which waived the requirement for informed consent.

\section{Patient consent for publication}

Not applicable.

\section{Competing interests}

The authors declare that they have no competing interests.

\section{References}

1. Bray F, Ferlay J, Soerjomataram I, Siegel RL, Torre LA and Jemal A: Global cancer statistics 2018: GLOBOCAN estimates of incidence and mortality worldwide for 36 cancers in 185 countries. CA Cancer J Clin 68: 394-424, 2018.

2. Krook JE, Moertel CG, Gunderson LL, Wieand HS, Collins RT, Beart RW, Kubista TP, Poon MA, Meyers WC and Mailliard JA, et al: Effective surgical adjuvant therapy for high-risk rectal carcinoma. N Engl J Med 324: 709-715, 1991.

3. Wolmark N, Wieand HS, Hyams DM, Colangelo L, Dimitrov NV, Romond EH, Wexler M, Prager D, Cruz AB Jr, Gordon PH, et al: Randomized trial of postoperative adjuvant chemotherapy with or without radiotherapy for carcinoma of the rectum: National surgical adjuvant breast and bowel project protocol R-02. J Natl Cancer Inst 92: 388-396, 2000.

4. Martling A, Holm T, Rutqvist LE, Johansson H, Moran BJ, Heald RJ and Cedermark B: Impact of a surgical training programme on rectal cancer outcomes in Stockholm. Br J Surg 92: 225-229, 2005.

5. van Gijn W, Marijnen CA, Nagtegaal ID, Kranenbarg EM, Putter H, Wiggers T, Rutten HJ, Påhlman L, Glimelius B and van de Velde CJ; Dutch Colorectal Cancer Group: Preoperative radiotherapy combined with total mesorectal excision for resectable rectal cancer: 12-Year follow-up of the multicentre, randomised controlled TME trial. Lancet Oncol 12: 575-582, 2011.

6. Sauer R, Becker H, Hohenberger W, Rödel C, Wittekind C, Fietkau R, Martus P, Tschmelitsch J, Hager E, Hess CF, et al: Preoperative versus postoperative chemoradiotherapy for rectal cancer. N Engl J Med 351: 1731-1740, 2004.

7. Sauer R, Liersch T, Merkel S, Fietkau R, Hohenberger W, Hess C, Becker H, Raab HR, Villanueva MT, Witzigmann H, et al: Preoperative versus postoperative chemoradiotherapy for locally advanced rectal cancer: Results of the German CAO/ARO/AIO-94 randomized phase III trial after a median follow-up of 11 years. J Clin Oncol 30: 1926-1933, 2012.

8. Song JH, Jeong JU, Lee JH, Kim SH, Cho HM, Um JW and Jang HS; Korean Clinical Practice Guideline for Colon and Rectal Cancer Committee: Preoperative chemoradiotherapy versus postoperative chemoradiotherapy for stage II-III resectable rectal cancer: A meta-analysis of randomized controlled trials. Radiat Oncol J 35: 198-207, 2017.

9. Li Y, Wang J, Ma X, Tan L, Yan Y, Xue C, Hui B, Liu R, Ma H and Ren J: A review of neoadjuvant chemoradiotherapy for locally advanced rectal cancer. Int J Biol Sci 12: 1022-1031, 2016.

10. Charlton ME, Lin C, Jiang D, Stitzenberg KB, Halfdanarson TR, Pendergast JF, Chrischilles EA and Wallace RB: Factors Associated with use of preoperative chemoradiation therapy for rectal cancer in the cancer care outcomes research and surveillance consortium. Am J Clin Oncol 36: 572-579, 2013.

11. Barker HE, Paget JT, Khan AA and Harrington KJ: The tumour microenvironment after radiotherapy: Mechanisms of resistance and recurrence. Nat Rev Cancer 15: 409-425, 2015.

12. Martin OA, Anderson RL, Narayan K and MacManus MP: Does the mobilization of circulating tumour cells during cancer therapy cause metastasis? Nat Rev Clin Oncol 14: 32-44, 2016.

13. Hugen N, van de Velde CJH, de Wilt JHW and Nagtegaal ID: Metastatic pattern in colorectal cancer is strongly influenced by histological subtype. Ann Oncol 25: 651-657, 2014.

14. Ding P, Liska D, Tang P, Shia J, Saltz L, Goodman K, Downey RJ, Nash GM, Temple LK, Paty PB, et al: Pulmonary recurrence predominates after combined modality therapy for rectal cancer. An original retrospective study. Ann Surg 256: 111-116, 2012. 
15. Oken MM, Creech RH, Tormey DC, Horton J, Davis TE, McFadden ET and Carbone PP: Toxicity and response criteria of the Eastern cooperative oncology group. Am J Clin Oncol 5: 649-655, 1982

16. Hamilton SR, Bosman FT, Boffetta P, Ilyas M, Morreau H, Nakamura SI, Quirke P, Riboli E and Sobin LH: Carcinoma of the colon and rectum: In: WHO Classification of Tumours of the Digestive System. Bosman FT, Carneiro F, Hruban RH and Theise ND (eds). IARC, Lyon, pp134-146, 2010.

17. Edge SB, Byrd SR, Compton CC, Fritz AG, Greene FL and Trotti A (eds.): AJCC Cancer Staging Manual. 7th edition. Springer-Verlag, New York, NY, pp143-164, 2010.

18. Hofheinz RD, Wenz F, Post S, Matzdorff A, Laechelt S, Hartmann JT, Müller L, Link H, Moehler M, Kettner E, et al: Chemoradiotherapy with capecitabine versus fluorouracil for locally advanced rectal cancer: A randomised, multicentre, non-inferiority, phase 3 trial. Lancet Oncol 13: 579-588, 2012.

19. Hong YS, Nam BH, Kim KP, Kim JE, Park SJ, Park YS, Park JO, Kim SY, Kim TY, Kim JH, et al: Oxaliplatin, fluorouracil, and leucovorin versus fluorouracil and leucovorin as adjuvant chemotherapy for locally advanced rectal cancer after preoperative chemoradiotherapy (ADORE): An open-label, multicentre, phase 2, randomised controlled trial. Lancet Oncol 15: 1245-1253, 2014.

20. Austin PC and Stuart EA: Moving towards best practice when using inverse probability of treatment weighting (IPTW) using the propensity score to estimate causal treatment effects in observational studies. Stat Med 34: 3661-3679, 2015.
21. Robins JM, Hernán MA and Brumback B: Marginal structural models and causal inference in epidemiology. Epidemiology 11: 550-560, 2000

22. Brookhart MA, Schneeweiss S, Rothman KJ, Glynn RJ, Avorn J and Stürmer T: Variable selection for propensity score models. Am J Epidemiol 163: 1149-1156, 2006.

23. Räsänen $M$, Carpelan-Holmström $M$, Mustonen $H$, Renkonen-Sinisalo L and Lepistö A: Pattern of rectal cancer recurrence after curative surgery. Int J Colorectal Dis 30: $775-785,2015$.

24. Segelman J, Akre O, Gustafsson UO, Bottai M and Martling A: Individualized prediction of risk of metachronous peritoneal carcinomatosis from colorectal cancer. Colorectal Dis 16: 359-367, 2014.

25. Sakuyama N, Kojima M, Kawano S, Akimoto T, Saito N, Ito M and Ochiai A: Histological differences between preoperative chemoradiotherapy and chemotherapy for rectal cancer: A clinicopathological study. Pathol Int 66: 273-280, 2016.

26. Nagtegaal I, Gaspar C, Marijnen C, van de Velde C, Fodde R and van Krieken H: Morphological changes in tumour type after radiotherapy are accompanied by changes in gene expression profile but not in clinical behaviour. J Pathol 204: 183-192, 2004.

27. Chiang JM, Hsieh PS, Chen JS, Tang R, You JF and Yeh CY: Rectal cancer level significantly affects rates and patterns of distant metastases among rectal cancer patients post curative-intent surgery without neoadjuvant therapy. World J Surg Oncol 12: 197, 2014. 